\title{
Numerical Studies of Resonance and Secular Effects of Gravitational Waves
}

\author{
M. H. A. Youssef \\ Astronomy Department, Faculty of Science, Cairo University, Cairo, Egypt \\ Email: mhyoussef@sci.cu.edu.eg
}

How to cite this paper: Youssef, M.H.A (2018) Numerical Studies of Resonance and Secular Effects of Gravitational Waves. World Journal of Mechanics, 8, 182-191. https://doi.org/10.4236/wjm.2018.85013

Received: March 17, 2018

Accepted: May 13, 2018

Published: May 16, 2018

Copyright $\odot 2018$ by author and Scientific Research Publishing Inc. This work is licensed under the Creative Commons Attribution International License (CC BY 4.0).

http://creativecommons.org/licenses/by/4.0/

\section{(c) (i) Open Access}

\begin{abstract}
This work deals with the numerical solution of the gravitational waves effects on the orbital elements of the planets in case of commensurability between the wave's frequency $n_{g}$ and the planet's mean motion $n_{p}$. Taking Mercury and Pluto as practical examples for low frequency and high frequency, the variations of the orbital elements of Mercury due to resonance of gravitational wave are different and small than the perturbation on Pluto. The amount of changing in the orbital elements under the effects of gravitational waves is different from planet to planet according to the planet's mean motion $n_{p}$. For low frequency $n_{g}$, the secular variation in orbital elements will be negative (i.e. decreasing) in the inclination, semi-major axis and the eccentricity $(i, a, e)$ like as Pluto. For high frequency $n_{g}$ like Mercury, the secular variation in all the orbital elements will be positive (i.e. increasing). The perturbation on all the orbital elements of two planets is changing during each revolution except the eccentricity $e$ of Mercury and the mean anomaly $M$ of Mercury and Pluto during the time.
\end{abstract}

\section{Keywords}

Gravitational Waves, Celestial Mechanics, Perturbation, Orbital Mechanics, Numerical Techniques

\section{Introduction}

All methods developed to detect gravitational waves depend more or less on the fact that the maximum variation in the particle separation occurs if the particles are located in XY-plane. The effect of gravitational waves is too small. A direct measurement of its physical properties is still lacking [1]. The gravitational waves spectrums cover an interval of about 18 orders of magnitude in wavelength [2]. The frequencies in the range $10-10^{4} \mathrm{~Hz}$ are the targets of several de- 
tectors like LIGO, TAMA, VIRGO, etc. Now the very low frequency is concerned $10^{-9}-10^{-7} \mathrm{~Hz}$ dealt with it [3]. Despite the wealth of the literature with works aiming at the evaluation of these effects, they are almost exclusively written from the viewpoint of physicists, with consequence that the powerful tools of celestial mechanics are still off. The effects of incident gravitational waves on the orbital motion of gravitational bound system were investigated analytically by several authors with different approaches and various feature of both orbits and the waves [4]-[13]. The idea of using the solar system to try to detect gravitational waves was first suggested by [14]. In the case of studying the effect of gravitational waves it is often useful to utilize the perturbative or disturbing function which is analogous to the potential function $U$ as in [15] [16] and [17]. The disturbing force of gravitational waves contains long, short and secular effects. Youssef (2017) has found that the effect of gravitational waves is pure short-period effects [15]. The short and long period's perturbations due to the effect of gravitational waves on the orbital elements of Jupiter are studies numerically [16]. The short and long period's perturbations due to the effect of gravitational waves on the orbital elements of planets for different sources of GW are studies in [17]. The typical planetary orbital frequencies vary from $1.3 \times 10^{-7} \mathrm{~Hz}$ for Mercury to $1.2 \times 10^{-10} \mathrm{~Hz}$ for Pluto. In this work we treat the effect of gravitational waves on orbital elements of Mercury of high mean motion and Pluto of low mean motion in case of the commensurability between the frequency of gravitational waves and the frequency of the planet using the Gauss form of Lagrange planetary equations. We determine the secular perturbations on the orbital elements using the Gauss form of Lagrange's equations. Numerical method of Runge-Kutta $4^{\text {th }}$ order is used to obtain the second order effect.

\section{Equations of Motion}

The wave creates a field of variable accelerations of the type

$$
\begin{aligned}
& F_{x}=h_{1} x+h_{2} y \\
& F_{y}=h_{2} x-h_{1} y \\
& F_{z}=0
\end{aligned}
$$

where $F_{x}, F_{y}$ and $F_{z}$ are the components of the acceleration vector of normal incident of plane gravitational wave in $(x, y, z)$ coordinates and

$$
h_{1}=\frac{1}{2} \frac{\partial^{2} h_{11}}{\partial t^{2}}, h_{2}=\frac{1}{2} \frac{\partial^{2} h_{12}}{\partial t^{2}}
$$

The two polarized components of the transverse GW are

$$
\begin{aligned}
& h_{11}=h_{+} \cos \left(n_{g} t+\alpha_{1}\right) \\
& h_{12}=h_{\times} \cos \left(n_{g} t+\alpha_{2}\right)
\end{aligned}
$$

where $n_{g}$ is the frequency of the wave, $\alpha_{1}$ and $\alpha_{2}$ are the phase difference, $h_{+}$and $h_{\times}$are the amplitude of the wave in the two orthogonal directions in the transverse plane. Substituting Equations (3) and (4) into (2) and then into (1), there- 
fore the acceleration components are

$$
\begin{gathered}
F_{x}=\beta \cos \left(n_{g} t+\alpha_{1}\right) x+\gamma \cos \left(n_{g} t+\alpha_{2}\right) y \\
F_{y}=\gamma \cos \left(n_{g} t+\alpha_{2}\right) x-\beta \cos \left(n_{g} t+\alpha_{1}\right) y \\
F_{z}=0
\end{gathered}
$$

where

$$
\beta=-\frac{1}{2} n_{g}^{2} h_{+} \text {and } \gamma=-\frac{1}{2} n_{g}^{2} h_{\times}
$$

Regarding the estimates of the values of the frequencies and amplitudes of gravitational waves from different sources, we can fairly assume that $\beta$ and $\gamma$ are of order the eccentricity of the elliptic orbit (e). Now we express the components of the acceleration in the directions $S, T, W$ along the unit vectors $P^{\wedge}, Q^{\wedge}$ and $W^{\wedge}$ in the direction of $r$, normal to $r$ in the orbital plane and normal to the orbital plane respectively as shown in Figure 1.

Therefore we have

$$
\begin{gathered}
S=r\left\{A_{1}+A_{2} \cos 2(f+\omega)+A_{3} \sin 2(f+\omega)\right\} \\
T=r\left\{-A_{2} \sin 2(f+\omega)+A_{3} \cos 2(f+\omega)\right\} \\
W=r\left\{B_{1} \cos (f+\omega)+B_{2} \sin (f+\omega)\right\} \sin i
\end{gathered}
$$

where

$$
\begin{gathered}
A_{1}=\frac{1}{2} \sin ^{2} i\left\{h_{1} \cos 2 \Omega+h_{2} \sin 2 \Omega\right\} \\
A_{2}=\frac{1+\cos ^{2} i}{2}\left\{h_{1} \cos 2 \Omega+h_{2} \sin 2 \Omega\right\} \\
A_{3}=\cos i\left\{-h_{1} \sin 2 \Omega+h_{2} \cos 2 \Omega\right\} \\
B_{1}=h_{1} \sin 2 \Omega-h_{2} \cos 2 \Omega \\
B_{2}=\cos i\left\{h_{1} \cos 2 \Omega+h_{2} \sin 2 \Omega\right\}
\end{gathered}
$$

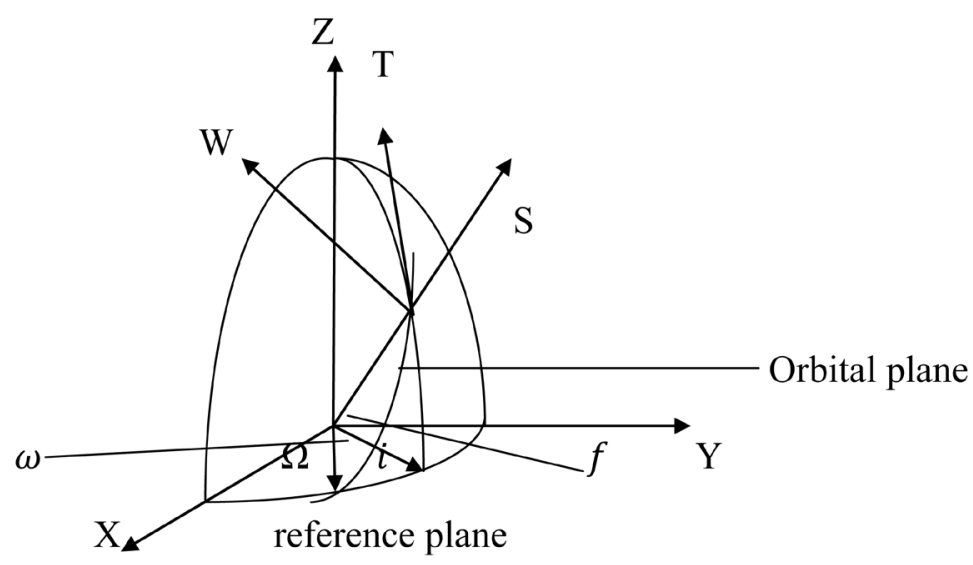

Figure 1. The disturbing force of gravitational waves in $(S, T, W)$ directions and the angle $i$ between the orbital plane and reference plane and the angles $(\Omega, \omega, f)$. 
$i, \omega$, and $f$ are the inclination, longitude of node, argument of perigee and the true anomaly of an orbit respectively. The Gauss form of Lagrange's planetary equations is (Roy, 1965) [18]

$$
\begin{gathered}
\frac{\mathrm{d} a}{\mathrm{~d} t}=\frac{2 e \sin f}{n \sqrt{1-e^{2}}} S+\frac{2 a \sqrt{1-e^{2}}}{n r} T \\
\frac{\mathrm{d} e}{\mathrm{~d} t}=\frac{\sqrt{1-e^{2}} \sin f}{n a} S+\frac{\sqrt{1-e^{2}}}{n a^{2} e} \frac{a n-r^{2}}{r} T \\
\frac{\mathrm{d} i}{\mathrm{~d} t}=\frac{r \cos (f+\omega)}{n a^{2} \sqrt{1-e^{2}}} W \\
\frac{\mathrm{d} \Omega}{\mathrm{d} t}=\frac{r \sin (f+\omega)}{n a^{2} \sqrt{1-e^{2}}} \sin i W \\
\frac{\mathrm{d} \omega}{\mathrm{d} t}=-\frac{\sqrt{1-e^{2}} \cos f}{n a e} S+\left(1+\frac{r}{p}\right) \frac{\sqrt{\left(1-e^{2}\right.}}{n a e} \sin f T-\frac{r \sin (f+\omega)}{n a^{2} \sqrt{1-e^{2}}} \cot i W \\
\frac{\mathrm{d} M}{\mathrm{~d} t}=n-\sqrt{1-e^{2}}\left\{-\frac{\sqrt{1-e^{2}} \cos f}{n a e} S+\left(1+\frac{r}{p}\right) \frac{\sqrt{1-e^{2}}}{n a e} \sin f T\right\}-\frac{2 r}{n a^{2}} S
\end{gathered}
$$

Substituting Equations (9), (10) and (11) into Equations (17) to (22). We solve these equations numerically using Runge-Kutta four order methods, the mathematical program written by language of MATHEMATICA V10. Considering the commensurability between the gravitational wave and the mean motion of Mercury and of Pluto (i.e. we are studying the effect of GW when the frequency of GW equal to the mean motion of Mercury and when the frequency of GW equal to the mean motion of Pluto).

\section{Solution and Results}

We now describe a perturbation approach to solve the above equations to yield the variation in the elements during any interval of time. The amount of perturbations depends on the orders of the disturbing forces such that when the perturbing force is small compared to $\frac{\mu}{r^{2}}$ we will not find large changes in the osculating elements like the force of gravitational waves, but this change may be not ignored for studying the gravitational waves effects in future. We describe a procedure to calculate the perturbations as numerical integration for the set of differential equations in the form

$$
\frac{\mathrm{d} X}{\mathrm{~d} t}=\epsilon f(x, t)
$$

where $\epsilon$ is a small parameter, then the solution will be in the form

$$
F^{K}=\sum_{j=1}^{k} \zeta^{j}+B
$$

$B$ is a constant n-vector and

$$
\zeta^{1}=\epsilon\left[\int f(x, t) \mathrm{d} t+C(x)\right]
$$




$$
\zeta^{j+1}=-\epsilon \int \zeta^{1} \cdot f(x, t) \mathrm{d} t, j=1,2
$$

where $\zeta_{x}^{j}$ is the Jacobian matrix of the set $\zeta^{j}$ with respect to the set $x, x$ is kept constant during the integration and $C(x)$ is an arbitrary function of $x$. The secular effects will obtain from (26), representing the second order effect. Using the elliptic orbit relations

$$
\begin{gathered}
\cos f=\frac{\cos E-e}{1-e \cos E} \\
\sin f=\frac{\sqrt{1-e^{2}} \sin E}{1-e \cos E} \\
r \cos f=a(\cos E-e) \\
r \sin f=a \sqrt{1-e^{2}} \sin E
\end{gathered}
$$

$E$ is the eccentric anomaly and related to the mean anomaly $M$ through the Kepler's equation

$$
E-e \sin E=M
$$

Changing the independent variable from the time $t$ to the eccentric anomaly $E$ and using the above elliptic relations in the equations of motion (17) to (22). Solving the equations numerically when the frequency of GW is equal to the frequency of Mercury and Pluto as in Table 1 and Table 2. Assuming $\alpha_{1}=-\frac{\pi}{2}$ and $\alpha_{2}=\frac{\pi}{2}$, and the amplitude is equal to $15 \times 10^{-21}$. The variation of the orbital elements during the time in radian due to the effect of GW for five revolutions is presented in Table 3 and Table 4 and in the Figure 2 and Figure 3.

Table 1. The orbital elements of mercury reference date 12.00 UT 1 Jan. 2000.

\begin{tabular}{cc}
\hline Elements of the Planet & Mercury \\
\hline The semi-major in Km $(a)$ & $57.9 \times 10^{6}$ \\
The eccentricity $(e)$ & 0.205627 \\
Inclination in degree $(i)$ & 7.00399 \\
Longitude of node in degree $\Omega$ & 47.85714 \\
Longitude of perihelion in degree $\omega$ & 76.83309 \\
Mean daily motion in degree/day $n$ & 4.092339 \\
\hline
\end{tabular}

Table 2. The orbital elements of Pluto reference date 12.00 UT 1 Jan. 2000.

\begin{tabular}{cc}
\hline Elements of the Planet & Pluto \\
\hline The semi-major in Km $(a)$ & $5896 \times 10^{6}$ \\
The eccentricity $(e)$ & 0.250236 \\
Inclination in degree $(i)$ & 17.1699 \\
Longitude of node in degree $\Omega$ & 109.88562 \\
Longitude of perihelion in degree $\omega$ & 224.16024 \\
Mean daily motion in degree/day $n$ & 0.003979 \\
\hline
\end{tabular}




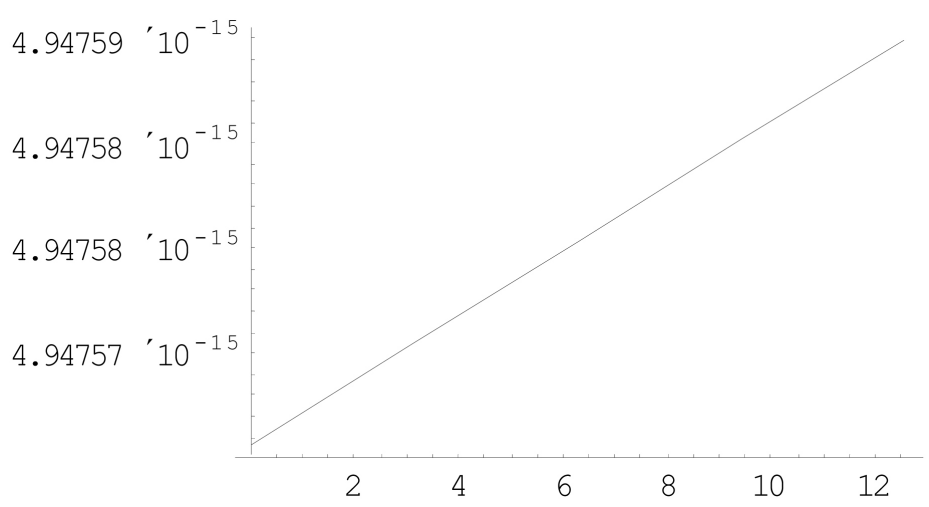

Variation of semi-major during five revolutions

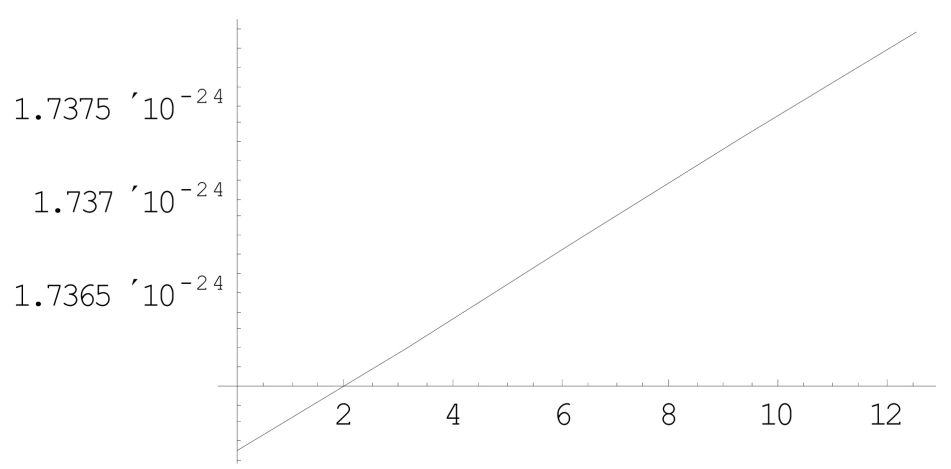

Variation of inclination during five revolutions
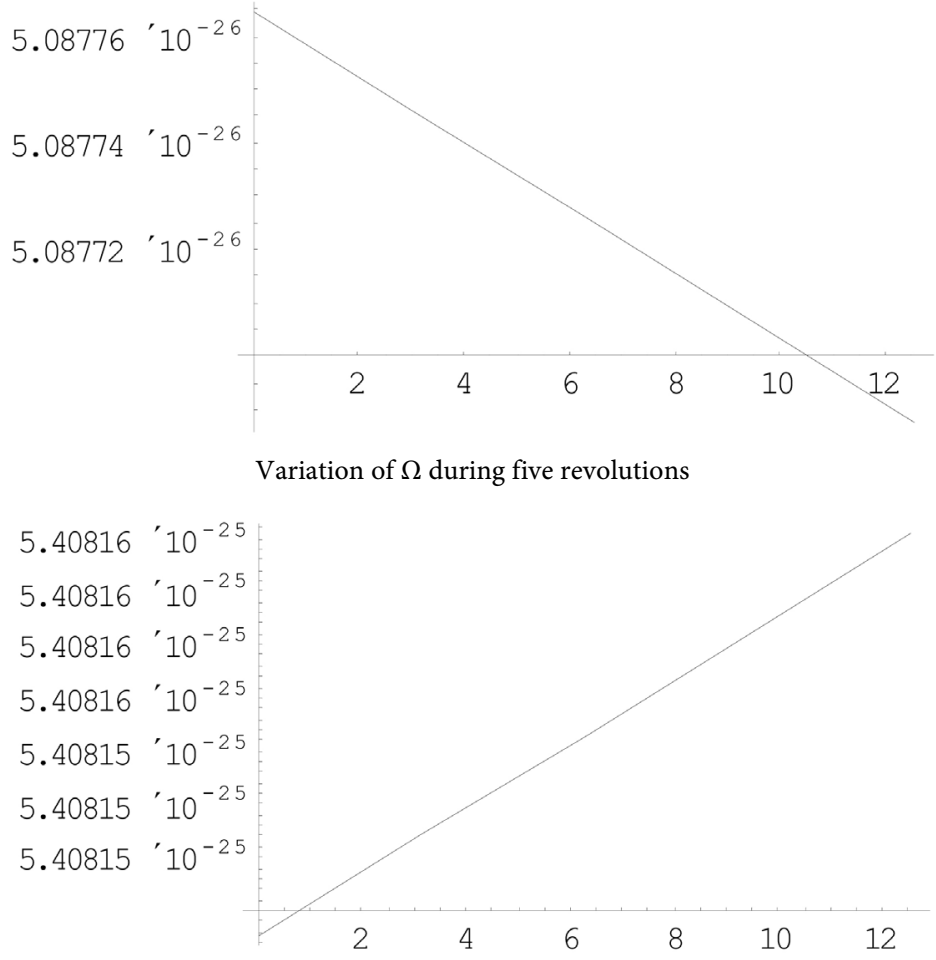

Variation of $\omega$ during five revolutions

Figure 2. The variation of the orbital elements of Mercury during five revolutions, X-axis represents the eccentric anomaly from 0 to $5 \pi$ and $\mathrm{Y}$-axis is the amount of variation of the orbital elements $(a, i, \Omega, \omega)$ in radian. 


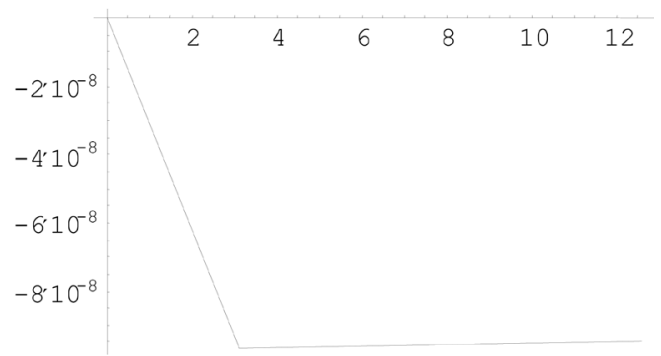

The variation of the semi-major during five revolutions

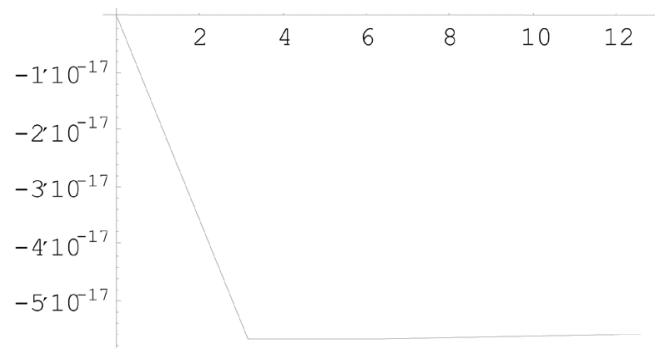

The variation of the eccentricity during five revolutions

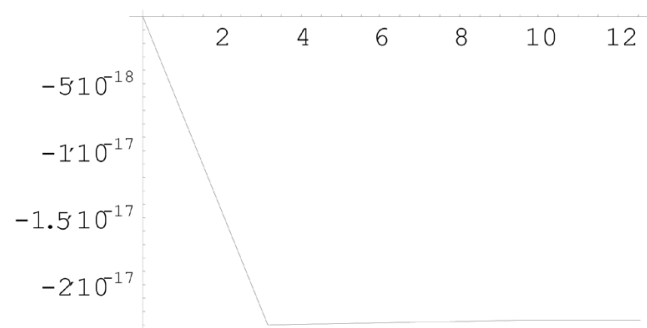

The variation of the inclination during five revolutions

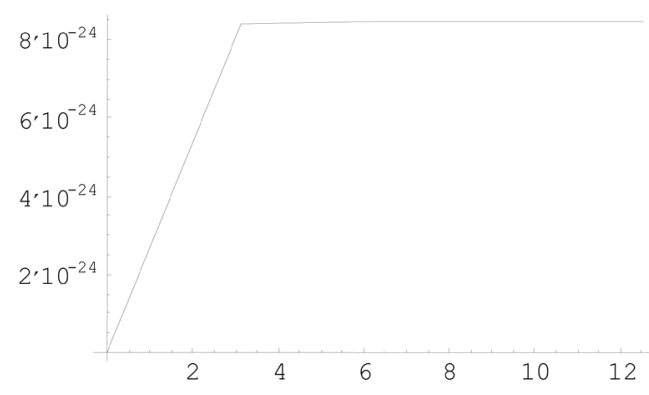

Variation of $\Omega$ during five revolutions

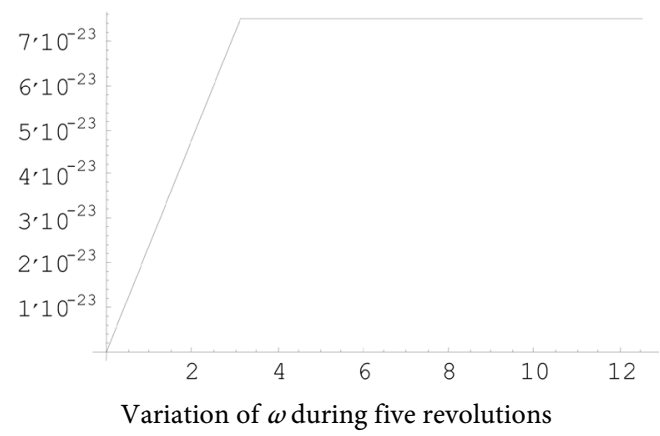

Figure 3. The variation of the orbital elements of Pluto during five revolutions, $\mathrm{X}$-axis represents the eccentric anomaly from 0 to $5 \pi$ and $\mathrm{Y}$-axis is the amount of variation of the orbital elements $(a, e, i, \Omega, \omega)$ in radian. 
Table 3. The variation of the elements of mercury due to the resonance of GW in radians.

\begin{tabular}{|c|c|c|c|}
\hline period & $\delta a$ & $\delta e$ & $\delta i$ \\
\hline 0 & $4.947566 \times 10^{\wedge}-15$ & $3.307374 \times 10^{\wedge}-22$ & $1.735647 \times 10^{\wedge}-24$ \\
\hline 1 & $4.947571 \times 10^{\wedge}-15$ & $3.307374 \times 10^{\wedge}-22$ & $1.736209 \times 10^{\wedge}-24$ \\
\hline 2 & $4.947575 \times 10^{\wedge}-15$ & $3.307374 \times 10^{\wedge}-22$ & $1.736770 \times 10^{\wedge}-24$ \\
\hline 3 & $4.947580 \times 10^{\wedge}-15$ & $3.307374 \times 10^{\wedge}-22$ & $1.737332 \times 10^{\wedge}-24$ \\
\hline 4 & $4.947585 \times 10^{\wedge}-15$ & $3.307374 \times 10^{\wedge}-22$ & $1.737894 \times 10^{\wedge}-24$ \\
\hline period & $\delta \omega$ & $\delta \Omega$ & $\delta M$ \\
\hline 0 & $5.408152 \times \wedge-25$ & $5.0877643 \times \wedge-26$ & $1.98933^{`} \times \wedge-7$ \\
\hline 1 & $5.408153 \times \wedge-25$ & $5.0877452 \times \wedge-26$ & $1.98933^{`} \times \wedge-7$ \\
\hline 2 & $5.408155^{`} \times \wedge-25$ & $5.0877261 \times \wedge-26$ & $1.98933^{`} \times \wedge-7$ \\
\hline 3 & $5.408157 \times \wedge-25$ & $5.0877070 \times \wedge-26$ & $1.98933^{`} \times \wedge-7$ \\
\hline 4 & $5.408159 \times \wedge-25$ & $5.0876879 \times \wedge-26$ & $1.98933^{`} \times \wedge-7$ \\
\hline
\end{tabular}

Table 4. The variation of the elements of Pluto due to the resonance of GW in radians.

\begin{tabular}{|c|c|c|c|}
\hline period & $\delta a$ & $\delta e$ & $\delta i$ \\
\hline 0 & 0 & 0 & 0 \\
\hline 1 & $-9.650559 \times \wedge-8$ & $-5.685610 \times \wedge-17$ & $-2.300079 \times \wedge-17$ \\
\hline 2 & $-9.582255 \times \wedge-8$ & $-5.650615 \times \wedge-17$ & $-2.288160 \times \wedge-17$ \\
\hline 3 & $-9.513952 \times \wedge-8$ & $-5.615620 \times \wedge-17$ & $-2.276240 \times \wedge-17$ \\
\hline 4 & $-9.445648 \times \wedge-8$ & $-5.580624 \times \wedge-17$ & $-2.264321 \times \wedge-17$ \\
\hline period & $\delta \omega$ & $\delta \Omega$ & $\delta M$ \\
\hline 0 & 0 & 0 & $0.0000167118^{\prime}$ \\
\hline 1 & $7.464780 \times \wedge-23$ & $8.438826 \times \wedge-24$ & $0.0000167118^{\prime}$ \\
\hline 2 & $7.464740 \times \wedge-23$ & $8.439177 \times \wedge-24$ & $0.0000167118^{`}$ \\
\hline 3 & $7.464700 \times \wedge-23$ & $8.439528 \times \wedge-24$ & $0.0000167118^{`}$ \\
\hline 4 & $7.464660 \times \wedge-23$ & $8.439879 \times \wedge-24$ & $0.0000167118^{\prime}$ \\
\hline
\end{tabular}

\section{Conclusion}

The resulting solution for our model from Equations (17) to (22) represents the secular effects of gravitational waves on the orbital elements under the commensurability of wave's frequency and the mean motion of the planets. The perturbations on the orbital elements of elliptic orbits are calculated numerically for five revolutions considering the time of perihelion passage is zero. The perturbation on semi-major a of Mercury increases with each revolution during the time from zero to $5 \pi$, but decreases for Pluto, and the perturbation on the eccentricity $e$ of Mercury did not change with revolution but decreasing for Pluto and the mean anomaly $M$ of Mercury and Pluto did not change as seen in Figure 2 and 
Figure 3. The amount of changing in the orbital elements under the effects of gravitational waves is different from planet to planet according to the planet's mean motion $n_{p}$. For low frequency $n_{g}$, the variation in orbital elements will be negative (i.e. decreasing) in the inclination, semi-major axis and the eccentricity $(i, a, e)$ like as Pluto. For high frequency $n_{g}$ like Mercury, the variation in all the orbital elements will be positive (i.e. increasing).

\section{References}

[1] Fairhurst, S., Guidi, G. and Hello, P. (2010) Current Status of Gravitational Wave Observation. General Relativity and Gravitation, 43, 387.

https://doi.org/10.1007/s10714-010-1009-1

[2] Prince, T.A. (2010) The Promise of Low-Frequency Gravitational Wave Astronomy for LISA International Science Team. The Astronomy and Astrophysics Decadal Survey, Science White Paper, No. 238.

[3] Flanagan, E. and Hughes, S. (2005) The Basics of Gravitational Wave Theory. New Journal of Physics, 7, 204. https://doi.org/10.1088/1367-2630/7/1/204

[4] Rudenko, V.N. (1975) Test Bodies under the Effect of Gravitational Radiation. Soviet Astronomy, 19, 270.

[5] Thorne, K.S. and Braginsky, V.B. (1976) Gravitational Wave Burst from the Nuclei of Distant Galaxies and Quasars. The Astrophysical Journal Letters, 204, L1. https://doi.org/10.1086/182042

[6] Mashoon, B. (1978) On Tidal Resonance. Astrophysical Journal, 223, 285. https://doi.org/10.1086/156262

[7] Mashoon, B. (1987) Wave Propagation in a Gravitational Field. Physics Letters A, 122, 299-304. https://doi.org/10.1016/0375-9601(87)90829-2

[8] Futamase, T. and Matsuda, T. (1979) Resonance between Primordial Gravitational Waves and Gravitationally Bound System. Progress of Theoretical Physics, 61, 86-93. https://doi.org/10.1143/PTP.61.86

[9] Turner, M.S. (1979) Influence of a Weak gravitational Wave on a Bound System of Two Point-Masses. Astrophysical Journal, 233, 685. https://doi.org/10.1086/157429

[10] Nelson, L.A. and Chau, W.Y. (1982) Orbital Perturbations of a Gravitationally Bound Two-Body System with the Passage of Gravitational Waves. Astrophysical Journal, 254, 735. https://doi.org/10.1086/159785

[11] Ivanshchenko, A.V. (1987) The Variation of the Keplerian Elements of a Planetary Orbit under the Action of a Gravitational Wave. Soviet Astronomy, 31, 76.

[12] Youssef, M.H. and Ahmed, M.K. (1997) Analytical Effects of Gravitational Waves on the Motion of an Artificial Satellite. Dynamics and Astrometry of Natural and Artificial Celestial Bodies, 431. https://doi.org/10.1007/978-94-011-5534-2_61

[13] Lorenzo, I. (2014) Orbital Effects of a Monochromatic Plane GW with Ultra-Low Frequency Incident on a Gravitationally Bound Two-Body System. ScienceOpen Research, V2, 1-13.

[14] Bertotti, B. (1973) Is the Solar System Gravitationally Closed? The Astrophysical Journal Letters, 14, 51.

[15] Youssef, M.H. (2017) Semi-Analytical Theory of the Mean Orbital Motion Due to the Effect of Gravitational Waves. European Journal of Scientific Research, 147, 342-350. 
[16] Youssef, M.H. (2017) Short-Period and Long-Period Effects of Weak Gravitational Waves. International Journal of Antimicrobial Agents, 7, 230-237.

https://doi.org/10.4236/ijaa.2017.73019

[17] Ismael, M.N. and Saad, N.A. (2011) The Effects of Gravitational Waves on the Orbital Elements of the Planets. The Open Astronomy Journal, 4, 1-5. https://doi.org/10.2174/1874381101104010001

[18] Roy, A.E. (1965) The Foundations of Astrodynamics. The Macmillan, New York. 